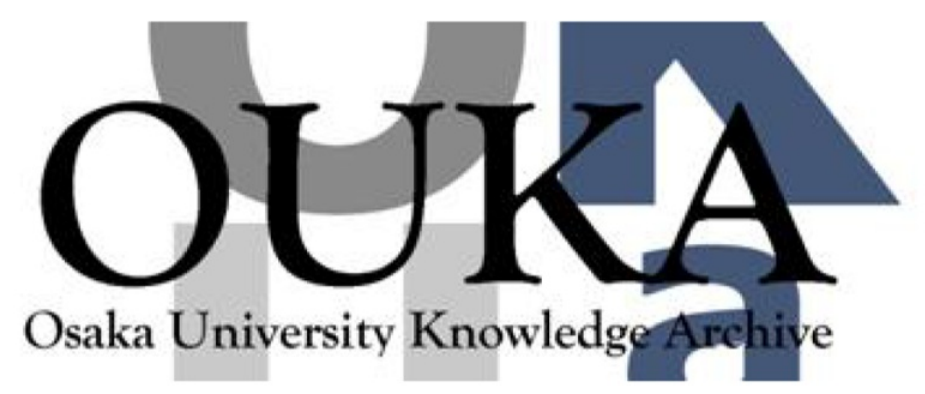

\begin{tabular}{|c|l|}
\hline Title & $\begin{array}{l}\text { Linearly polarized lasing in one-dimensional } \\
\text { hybrid photonic crystal containing cholesteric } \\
\text { Liquid crystal }\end{array}$ \\
\hline Author(s) & $\begin{array}{l}\text { Matsuhisa, Yuko; Ozaki, Ryotaro; Takao, Yuuki } \\
\text { et al. }\end{array}$ \\
\hline Citation & $\begin{array}{l}\text { Journal of Applied Physics. 101(3) p. 033120- } \\
\text { p. 033120 }\end{array}$ \\
\hline Issue Date & $2007-02-12$ \\
\hline oaire:version & VoR \\
\hline URL & https://hdl.handle. net/11094/75847 \\
\hline rights & \\
\hline Note & \\
\hline
\end{tabular}

Osaka University Knowledge Archive : OUKA

https://ir. Library. osaka-u. ac. jp/

Osaka University 


\section{Linearly polarized lasing in one-dimensional hybrid photonic crystal containing cholesteric liquid crystal}

Cite as: J. Appl. Phys. 101, 033120 (2007); https://doi.org/10.1063/1.2434835

Submitted: 25 July 2006 . Accepted: 05 December 2006 . Published Online: 12 February 2007

Yuko Matsuhisa, Ryotaro Ozaki, Yuuki Takao, and Masanori Ozaki

\section{ARTICLES YOU MAY BE INTERESTED IN}

High $Q$ defect mode and laser action in one-dimensional hybrid photonic crystal containing cholesteric liquid crystal

Applied Physics Letters 89, 101109 (2006); https://doi.org/10.1063/1.2347114

Low-threshold and high efficiency lasing upon band-edge excitation in a cholesteric liquid crystal

Applied Physics Letters 90, 091114 (2007); https://doi.org/10.1063/1.2710777

How doping a cholesteric liquid crystal with polymeric dye improves an order parameter and makes possible low threshold lasing

Journal of Applied Physics 94, 279 (2003); https://doi.org/10.1063/1.1578534

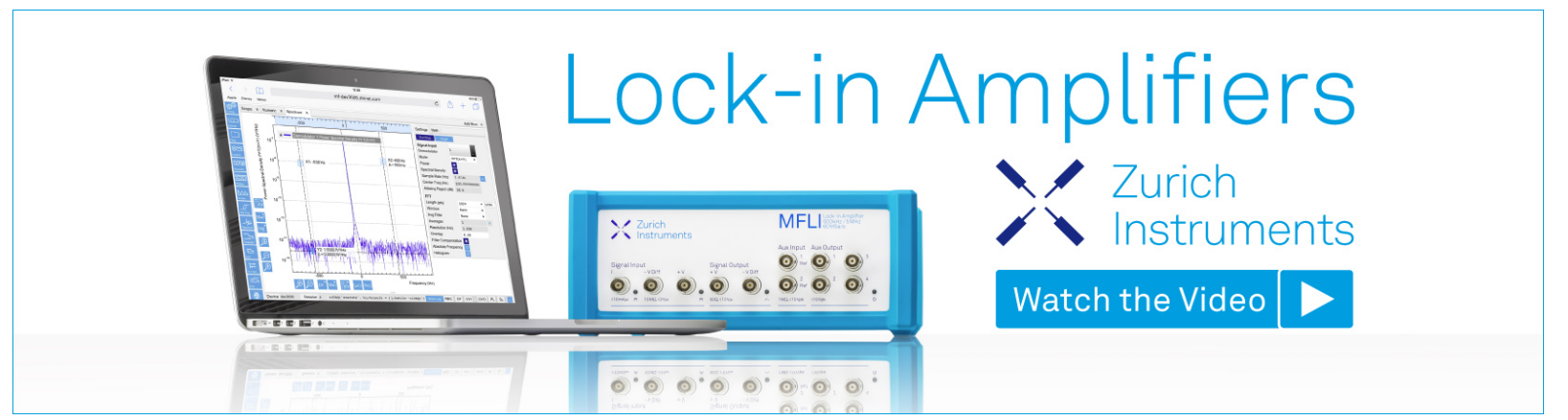

J. Appl. Phys. 101, 033120 (2007); https://doi.org/10.1063/1.2434835 


\title{
Linearly polarized lasing in one-dimensional hybrid photonic crystal containing cholesteric liquid crystal
}

\author{
Yuko Matsuhisa, Ryotaro Ozaki, Yuuki Takao, and Masanori Ozakia) \\ Department of Electrical, Electronic and Information Engineering, Graduate School of Engineering, \\ Osaka University, 2-1 Yamada-oka, Suita, Osaka 565-0871, Japan
}

(Received 25 July 2006; accepted 5 December 2006; published online 12 February 2007)

\begin{abstract}
We have achieved single-mode laser action in a one-dimensional hybrid photonic crystal (HPC) containing cholesteric liquid crystal (CLC) as a defect and experimentally investigated the polarization characteristics of the laser light. The laser light emitted from the HPC containing the CLC was linearly polarized, which was different from that of the simple CLC, despite the CLC being contained in this structure. We have theoretically calculated the optical propagation to justify the experimental result. The calculated result was in good agreement with the experimental result and showed the difference in optical characteristics between the simple CLC and the HPC containing the CLC. () 2007 American Institute of Physics. [DOI: 10.1063/1.2434835]
\end{abstract}

\section{INTRODUCTION}

Photonic crystals (PCs) have a periodic dielectric structure with a periodicity in a range of optical wavelengths. They have attracted much attention from both theoretical and practical points of view because of their potential for controlling light. In PCs, the propagation of light is inhibited in a certain energy range of photons, which results in the appearance of a photonic band gap (PBG). ${ }^{1}$ Furthermore, photons are localized upon the introduction of a defect in PCs. ${ }^{2}$ By utilizing the photon localization, various applications are expected. ${ }^{3-6}$

On the other hand, cholesteric liquid crystals (CLCs) and chiral smectic liquid crystals have chirality in their molecular structures and spontaneously form one-dimensional (1D) periodic helical structures in an optical range, which are regarded as 1D PCs. In CLCs with a helical structure, circularly polarized light with the same handedness as the helix propagating along a helical axis is selectively reflected, and a stop band appears. At the edge of the stop band, only the circularly polarized light with the same handedness as the helix effectively interacts with the medium of the CLC because photon group velocity is suppressed. By utilizing such an optical characteristic, circularly polarized laser actions have been achieved in dye-doped chiral liquid crystals. ${ }^{7-11}$

We have investigated hybrid photonic crystals (HPCs), which are composed of an inorganic PC and liquid crystal, and have proposed a tunable PC, in which a PBG and photon localization can be controlled. ${ }^{12-18}$ Recently, we have investigated a 1D HPC containing a CLC defect and have achieved low-threshold single-mode lasing based on a particular defect mode at the edge of the PBG of the CLC, which is based on the double optical confinement in the periodic structures of both the inorganic $\mathrm{PC}$ and the CLC. ${ }^{19}$ In this report, we investigate the polarization characteristics of the laser light in a 1D HPC containing the CLC as a defect. Furthermore, we determine the characteristics of optical propagation in this structure by theoretical calculation.

\section{EXPERIMENT}

A schematic diagram of a 1D HPC containing a CLC defect is shown in Fig. 1. The 1D HPC containing a CLC defect was fabricated with a double periodic structure of the CLC and dielectric multilayers. The dielectric multilayers, consisting of five pairs of alternately stacked $\mathrm{SiO}_{2}$ and $\mathrm{TiO}_{2}$ layers deposited on a glass substrate, were fabricated on both sides of the CLC. The refractive indices of $\mathrm{SiO}_{2}$ and $\mathrm{TiO}_{2}$ were 1.46 and 2.35 , and the thicknesses of their layers were 111 and $69 \mathrm{~nm}$, respectively. The center wavelength of the PBG of the multilayer was $650 \mathrm{~nm}$. The top surface of the dielectric multilayer was coated with polyimide (JSR, AL1254) and unidirectionally rubbed toward the $y$ axis to realize a planar alignment, in which the helix axis is perpendicular to the substrates.

The CLC host with a left-handed helix was prepared by mixing an optically active agent with a chiral center (Merck, S-811) and a nematic liquid crystal (Merck, E44). As a laser dye dopant, [2-(2-4(dimethylamino)pheny1)etheny1]-6methy1-4H-pyran-4-ylidene propanedinitrile (Exciton, DCM) was compounded at $1 \mathrm{wt} \%$ concentration in the CLC. The extraordinary and ordinary refractive indices of the CLC were 1.72 and 1.54 , respectively, and the PBG was within that of the multilayer. The CLC was induced between dielectric multilayers with $8.7 \mu \mathrm{m}$ spacing. In order to make

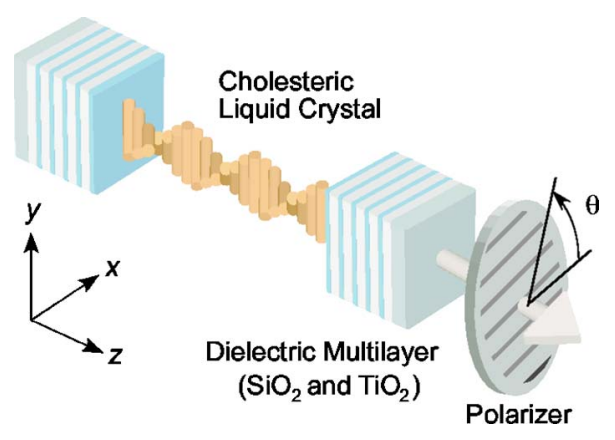

FIG. 1. 1D HPC containing CLC as defect.

${ }^{a)}$ Electronic mail: ozaki@eei.eng.osaka-u.ac.jp 

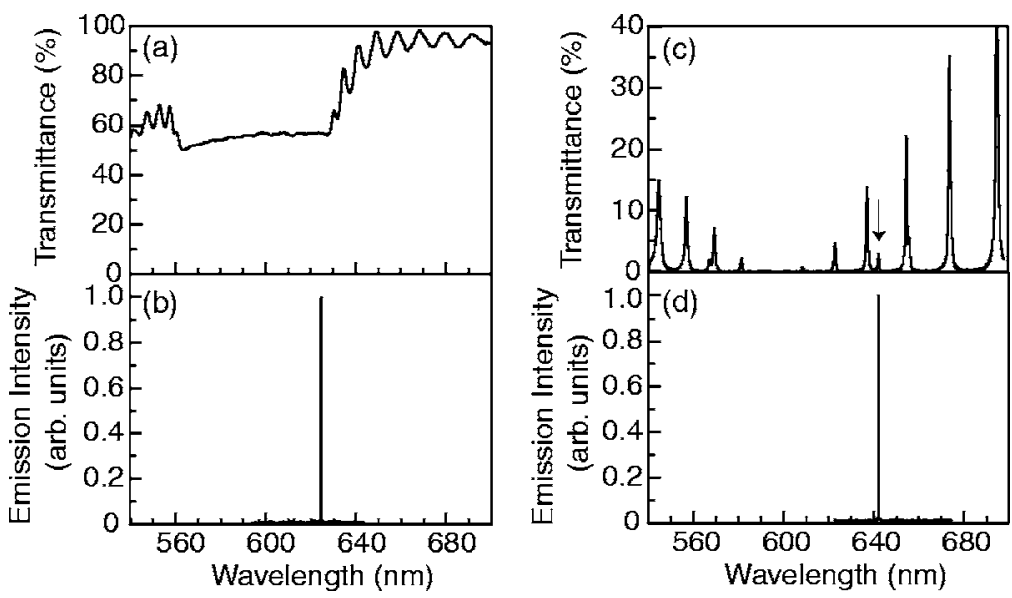

FIG. 2. (a) Transmission spectrum of CLC. (b) Emission spectrum of CLC. (c) Transmission spectrum of HPC containing CLC. (d) Emission spectrum of HPC containing CLC. a comparison, a cell of simple CLC sandwiched by glass substrates without multilayers was also composed in the same way.

We investigated the laser action in the simple CLC and the HPC containing a CLC defect. The second harmonic light of a $Q$-switch Nd:YAG (yttrium aluminum garnet) laser (Spectra Physics, Quanta-Ray INDI) was used for the excitation of the doped dye in the CLC, whose wavelength, pulse width, and pulse repetition frequency were $532 \mathrm{~nm}, 8 \mathrm{~ns}$, and $10 \mathrm{~Hz}$, respectively. The excitation laser beam irradiated the sample over an irradiated area of $0.2 \mathrm{~mm}^{2}$. The emitting light from the sample passed through a polarizer and was measured using a multichannel spectrometer (Oriel, MS257).

\section{EXPERIMENTAL RESULTS}

Figures 2(a) and 2(b) show the transmission spectrum and emission spectrum, respectively, at the pumping energy of $124 \mathrm{~nJ} /$ pulse for the simple CLC without multilayers. A decrease in transmittance due to the PBG was observed in the spectral range from 563 to $625 \mathrm{~nm}$. When the cell was excited above the threshold, single-mode laser action was observed at the wavelength of $625 \mathrm{~nm}$, as shown in Fig. 2(b). The lasing wavelength corresponded to the long-wavelength edge of the PBG, which was attributed to the suppression of photon group velocity at the edge of the PBG.

Figures 2(c) and 2(d) show the transmission spectrum and emission spectrum at the pumping energy of $36 \mathrm{~nJ} /$ pulse for the HPC containing the CLC defect. The outer inorganic $\mathrm{PC}$ has a PBG in the spectral range between 540 and 787 nm; therefore, Fig. 2(c) shows a partial spectral range of the transmission spectrum within the PBG of the PC. Many peaks were observed at regular intervals in Fig. 2(c), which correspond to defect modes resulting from the introduction of the CLC in the PC. However, one additional peak, indicated with the arrow in Fig. 2(c), was observed at $642 \mathrm{~nm}$, which corresponds to the band-edge wavelength of the CLC. From detailed consideration of the polarization states of transmitted light, the additional peak was clearly distinguished from the other defect mode peaks. Such a peak was not observed in a 1D PC with a uniform defect such as an isotropic medium or nematic liquid crystals. Namely, this peak is a defect mode peculiar to the helix defect in the 1D $\mathrm{PC}$ and is associated with photon localization originating from the band-edge effect of the CLC helix. When the HPC was excited above the threshold, single-mode laser action was observed which is based on the particular defect mode at the edge of the PBG of the CLC at the wavelength of $642 \mathrm{~nm}$. This is attributed to the double optical confinement of the band-edge effect of the CLC and the photon localization in the defect of the 1D PC composed with multilayers. ${ }^{19}$ The difference in lasing wavelength of the simple CLC and the HPC containing CLC observed is attributed to the measured temperature difference.

In order to investigate the polarization of the laser light of the simple CLC and the HPC containing the CLC defect, we measured the emission intensity of laser light as the polarizer rotated. The white squares and black circles in Fig. 3 show the emission intensity of the simple CLC and the HPC as a function of polarizer orientation. In the case of the CLC, the emission intensity was constant, which indicates that the laser light of the CLC was circularly polarized. At the edge of the PBG, a circularly polarized light with the same handedness as the helix of the CLC effectively interacted with the medium of the CLC because photon group velocity was suppressed. Hence, the laser light in the CLC was circularly polarized. On the other hand, the emission intensity of the HPC changed sinusoidally. It was maximized at $0^{\circ}$, which corresponds to the $x$ axis, and minimized to zero at $90^{\circ}$, which corresponds to the $y$ axis. This result indicates that the laser light was linearly polarized parallel to the $x$ axis, which

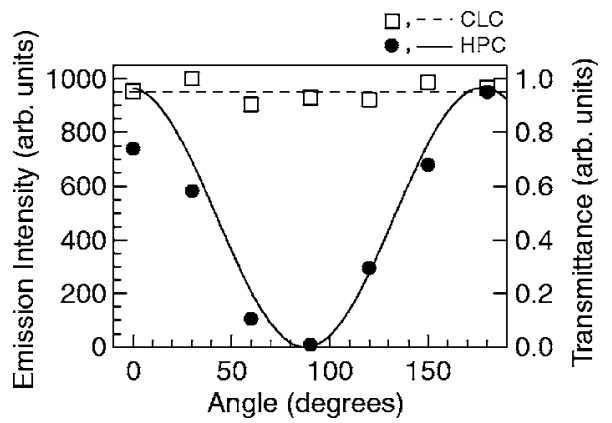

FIG. 3. Experimental emission intensity of simple CLC (white square) and HPC containing CLC defect (black circle) as a function of polarizer orientation. Calculated transmission intensity of simple CLC (dashed line) and HPC containing CLC defect (solid line) as a function of polarizer orientation. 


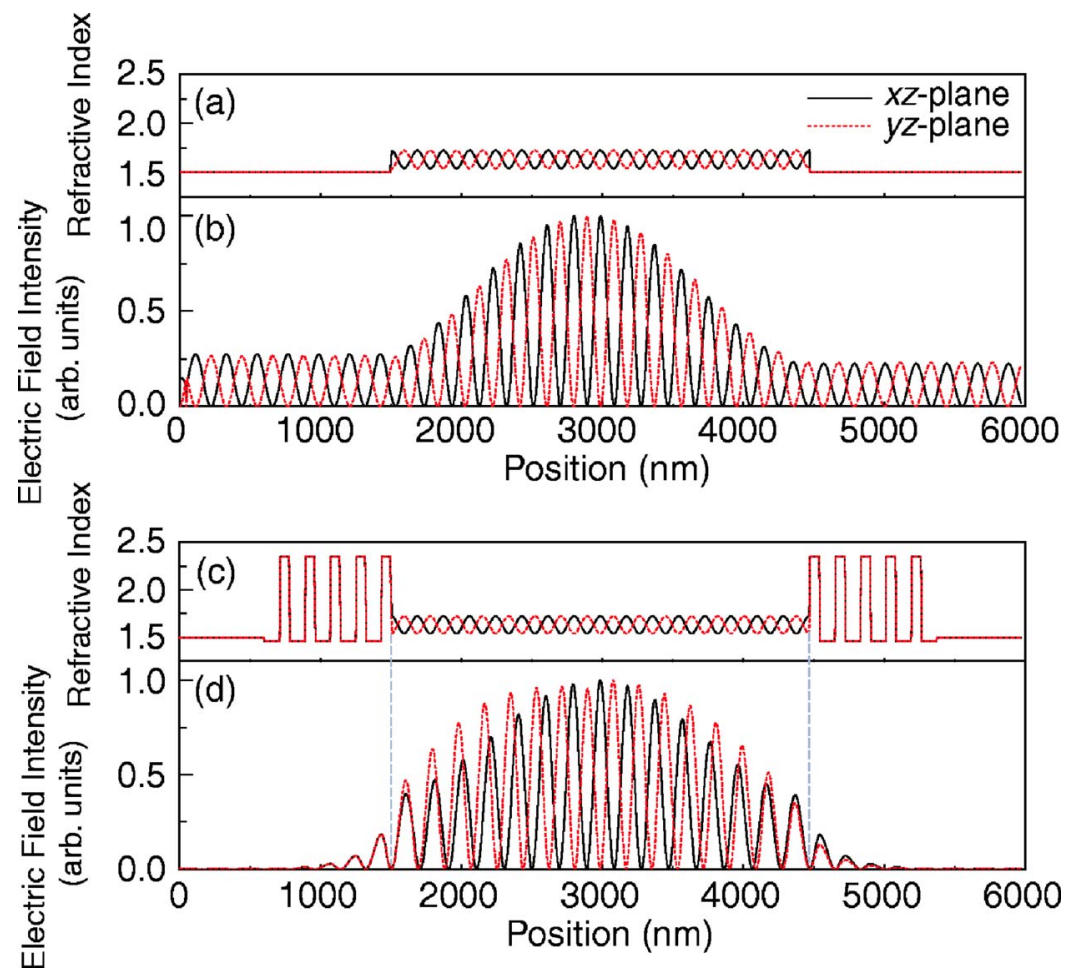

FIG. 4. (a) Distributions of refractive index of CLC. (b) Distributions of electric field intensity of CLC. (c) Distributions of refractive index of 1D HPC containing CLC. (d) Distributions of electric field intensity of 1D HPC containing CLC in $x z$ plane (solid line) and $y z$ plane (dashed line).

was different from the case of the simple CLC. Note that the laser light from the HPC containing the CLC was linearly polarized, despite the CLC being contained in this system. The laser light from the simple CLC should generally be circularly polarized, as shown by the white squares in Fig. 3 . However, when the CLC is sandwiched between dielectric multilayers, the laser light at the particular defect mode at the band edge of CLC becomes linearly polarized.

\section{CALCULATION}

To confirm the polarization of the laser light, we performed theoretical calculations using characteristic matrices. ${ }^{20}$ This is a method of numerical analysis based on Maxwell's equations. In this method, the light propagating along the $z$ axis with frequency $\omega$ is given by

$$
\frac{d \boldsymbol{\Psi}(z)}{d z}=\frac{i \omega}{c} \mathbf{D}(z) \boldsymbol{\Psi}(z),
$$

where $\mathbf{D}(z)$ is a derivative propagation matrix and $\boldsymbol{\Psi}(z)$ $=\left(E_{x}, H_{y}, E_{y}, H_{x}\right)^{T}$. By utilizing this method, we calculated the transmission intensity through the simple CLC at the band edge of CLC or the HPC at the particular defect mode and the polarizer as a function of polarized orientation.

The dashed line in Fig. 3 shows the calculated transmission intensity through the simple CLC and the polarizer at the longer edge of the PBG as a function of polarizer orientation. The transmission intensity was constant, which is in good agreement with the experimental result. On the other hand, the solid line shows the polarizer orientation dependence of the calculated transmission intensity through the HPC and the polarizer at the defect mode wavelength, at which the single-mode laser action was observed, as shown in Fig. 2(d). The transmission intensity changed sinusoidally and became zero at an angle of $88^{\circ}$, which shows good agreement with the experimental result. From these results, it was demonstrated both experimentally and theoretically that the laser light in a HPC containing a CLC defect was linearly polarized, despite the CLC being contained in the structure.

In order to clarify the reason for linearly polarized laser light being generated in the HPC containing the CLC, we calculated the electric field distributions of the simple CLC and the HPC using a finite-difference time domain (FDTD) method. This is a method of numerical analysis based on Maxwell's equations in differential form. In this calculation, we assumed the same refractive index distribution as that of the experimental setup, except for the thickness of the CLC defect. We used a CLC defect with $8.7 \mu \mathrm{m}$ thickness in the experiment; however, the calculated result under the same conditions as the experiment was complicated. Therefore, we show a simplified calculated result using a CLC defect with $3.0 \mu \mathrm{m}$ thickness. The result was essentially the same as the result of using a CLC defect with $8.7 \mu \mathrm{m}$ thickness.

Figures 4(a) and 4(b) show the distributions of the refractive index and the electric field intensity, respectively, of the simple CLC in the $x z$ and $y z$ planes at the wavelength of the longer edge of the PBG. Each refractive index in the $x z$ and $y z$ planes changed sinusoidally along the $z$ axis. However, the phases had a difference of $\pi / 2$ because of anisotropy and the helical alignment of the CLC molecules. The electric fields formed standing waves and were localized at each part of the high (extraordinary) refractive index. As a result, the electric field distribution in the $x z$ plane also shifted by $\pi / 2$ relative to that in the $y z$ plane, which indi- 
cates the generation of circularly polarized light. This wave profile is the typical field pattern at the longer edge of the PBG in the simple CLC and corresponds to the experimental result.

Figures 4(c) and 4(d) show the distributions of the refractive index and the electric field intensity, respectively, of the HPC containing the CLC defect in the $x z$ and $y z$ planes at the wavelength of the defect mode at the longer edge of the PBG of the CLC. At the middle of the CLC layer, the electric fields show the same distributions as the simple CLC, as shown in Fig. 4(b), which indicates that the electric field distribution in the $x z$ plane shifted by $\pi / 2$ relative to that in the $y z$ plane. However, the phase difference between the $x z$ and $y z$ planes decreased approaching the interfaces between the CLC and the multilayers (positions: 1500 and $4470 \mathrm{~nm}$ ). Consequently, the electric fields overlapped and became in phase at the boundaries, which was different from the case of the simple CLC. This change should result from a boundary condition. $^{21}$ In order to form standing waves, the electric fields must have nodes at the interfaces, as shown with dashed gray lines in Fig. 4(d), which causes the electric fields in the $x y$ and $y z$ planes to be in phase. Hence, the outgoing light became linearly polarized, which was in good agreement with the experimental result.

\section{CONCLUSION}

We achieved single-mode laser actions in a 1D HPC containing a CLC defect. We both experimentally and theoretically investigated the polarization of the laser light. The polarization of the laser light emitted from the HPC containing the CLC was linearly polarized, which was different from the simple CLC, despite the CLC being contained in the HPC. We performed the theoretical calculation of the optical propagation and showed the origin of the linearly polarized light generated in the HPC containing the CLC.

\section{ACKNOWLEDGMENT}

This work is partially supported by a Grant-in-Aid for Scientific Research from the Ministry of Education, Culture, Sports, Science and Technology of Japan.

${ }^{1}$ E. Yablonovitch, Phys. Rev. Lett. 58, 2059 (1987).

${ }^{2}$ S. John, Phys. Rev. Lett. 58, 2486 (1987).

${ }^{3}$ J. S. Foresi et al., Nature (London) 390, 143 (1997).

${ }^{4}$ O. Painter, R. K. Lee, A. Scherer, A. Yariv, J. D. O’Brien, P. D. Dapkus, and I. Kim, Science 284, 1819 (1999).

${ }^{5}$ S. Noda, A. Chutinan, and M. Imada, Nature (London) 407, 608 (2000).

${ }^{6}$ A. Chutinan and S. John, Phys. Rev. E 71, 026605 (2005).

${ }^{7}$ V. I. Kopp, B. Fan, H. K. M. Vithana, and A. Z. Genack, Opt. Lett. 23, 1707 (1998)

${ }^{8}$ B. Taheri, A. F. Munoz, P. Palffy-Muhoray, and R. Twieg, Mol. Cryst. Liq. Cryst. Sci. Technol., Sect. A 358, 73 (2001).

${ }^{9}$ A. F. Munoz, P. Palffy-Muhoray, and B. Taheri, Opt. Lett. 26, 804 (2001).

${ }^{10}$ T. Matsui, R. Ozaki, K. Funamoto, M. Ozaki, and K. Yoshino, Appl. Phys. Lett. 81, 3741 (2002).

${ }^{11}$ K. Funamoto, M. Ozaki, and K. Yoshino, Jpn. J. Appl. Phys., Part 2 42, L1523 (2003).

${ }^{12}$ K. Yoshino, S. Satoh, Y. Shimoda, Y. Kawagishi, K. Nakayama, and M. Ozaki, Jpn. J. Appl. Phys., Part 1 38, 961 (1999).

${ }^{13}$ K. Yoshino, Y. Shimoda, Y. Kawagishi, K. Nakayama, and M. Ozaki, Appl. Phys. Lett. 75, 932 (1999).

${ }^{14}$ Y. Shimoda, M. Ozaki, and K. Yoshino, Appl. Phys. Lett. 79, 3627 (2001).

${ }^{15}$ R. Ozaki, T. Matsui, M. Ozaki, and K. Yoshino, Jpn. J. Appl. Phys., Part 2 41, L1482 (2002).

${ }^{16}$ R. Ozaki, T. Matsui, M. Ozaki, and K. Yoshino, Appl. Phys. Lett. 82, 3593 (2003).

${ }^{17}$ R. Ozaki, M. Ozaki, and K. Yoshino, Jpn. J. Appl. Phys., Part 2 42, L669 (2003).

${ }^{18}$ R. Ozaki, Y. Matsuhisa, M. Ozaki, and K. Yoshino, Appl. Phys. Lett. 84, 1844 (2004).

${ }^{19}$ Y. Matsuhisa, R. Ozaki, M. Ozaki, and K. Yoshino, Jpn. J. Appl. Phys., Part 2 44, L629 (2005).

${ }^{20}$ D. W. Berreman, J. Opt. Soc. Am. 62, 502 (1972).

${ }^{21}$ E. Hecht, Optics, 4th ed. (Addison-Wesley, Reading, MA, 2002). 